\title{
The added value of chemical shift imaging in evaluation of bone marrow changes in sickle cell disease
}

\author{
Mohammad Fouad Abdel-Baki Allam(D, Manal Fayez Abu Samra and Al Shaimaa Mahfouz Abdel Rahman
}

\begin{abstract}
Background: The aim of this study was to assess the added value of chemical shift imaging when used with routine MRI study in evaluation of bone marrow changes in SCD. Forty-two patients with SCD and bone pain were included in the study; they underwent CSI and routine MRI study on the symptomatic anatomic part of the skeleton.

Results: Four patterns of diffuse bone marrow changes were recognized; they varied from persistent red marrow to diffuse hypointense patterns with abnormal signal loss percentage on CSI that suggest presence of iron overload ( $n$ $=28,66.6 \%)$. Serum ferritin level was increasing in accordance to the degree of signal changes found on CSI with significant high negative correlation between the percentage of signal loss on CSI obtained from IP-OP/IP formula and serum ferritin level. In focal marrow lesions, all T1 hyperintense lesions demonstrated corresponding hyperintensity on IP and OP; the detection frequency on CSI was relatively higher on OP compared with IP images.

Conclusion: CSI has high diagnostic performance in detecting diffuse marrow changes and development of iron overload in SCD. In SCD-related focal marrow lesions, CSI could have a complementary role in detection of T1 hyperintensity and lesion conspicuity.
\end{abstract}

Keywords: In phase, Out of phase, Chemical shift imaging, Bone marrow, Sickle cell disease

\section{Background}

Sickle cell disease (SCD) is one of the hemoglobinopathies that is characterized by abnormal production of hemoglobin, repeated cycles of sickling and unsickling, stasis and sequestration of blood cells, vascular obstruction, red cell destruction, hemolytic anemia, and bone marrow hyperplasia. SCD can result in persistence of hematopoietic (red) marrow within the appendicular skeleton; moreover, it can cause various skeletal problems that include osteomyelitis and progressive bone infarctions which may occur anywhere in the skeleton and are the source of painful bone crises [1,2].

As blood transfusion has a prominent role in patient's management in SCD, there is an increased risk of

\footnotetext{
* Correspondence: mfallam@mu.edu.eg

Faculty of Medicine, Minia University, Minia, Egypt
}

development of significant iron overload and subsequent iron-related complications to many organs [3].

Magnetic resonance imaging (MRI) is the most effective imaging tool used to evaluate focal bone marrow abnormalities as well as transfusion-related iron overload in a noninvasive way. Iron overload detection on MRI could be attributed to the susceptibility effect of iron which produces loss of signals in the involved tissues; the most common utilized sequence is the T2*-weighted sequence. Dual-sequence T1 gradient in-phase (IP) and out-of-phase (OP) imaging (a.k.a. chemical shift imaging (CSI)) can be used in the same manner to evaluate iron overload. Being characterized by higher echo time than the OP images, the IP images are more sensitive to iron deposition because of its $\mathrm{T}^{*}$ effect, this in turn explains why signal intensity reduction of iron-overloaded marrow is observed on IP not OP images [4, 5].

\section{Springer Open}

(ㅇ The Author(s). 2021 Open Access This article is licensed under a Creative Commons Attribution 4.0 International License, which permits use, sharing, adaptation, distribution and reproduction in any medium or format, as long as you give appropriate credit to the original author(s) and the source, provide a link to the Creative Commons licence, and indicate if changes were made. The images or other third party material in this article are included in the article's Creative Commons licence, unless indicated otherwise in a credit line to the material. If material is not included in the article's Creative Commons licence and your intended use is not permitted by statutory regulation or exceeds the permitted use, you will need to obtain permission directly from the copyright holder. To view a copy of this licence, visit http://creativecommons.org/licenses/by/4.0/. 


\section{Aim of the work}

The aim of this study was to assess the added value of chemical shift imaging when used with the routine MRI study in evaluation of bone marrow changes in sickle cell disease.

\section{Methods}

This observational analytic prospective study was conducted from February 2019 through December 2019 after being ethically approved by the institution committee.

\section{Study participants}

This study included forty two (twenty males and twentytwo females) patients known to have SCD. All patients were complaining of bone pain when they were referred from the emergency room and/or orthopedic department to the MRI unit. They underwent chemical shift imaging (T1-weighted dual gradient-echo in-phase and out-of-phase sequence) which was included within the routine MRI study on the symptomatic anatomic part of the skeleton. All the patients underwent complete clinical examination, CBC, differential white blood cell count, inflammatory markers, serum ferritin, and renal function tests. Informed written consent was obtained from all patients prior to study.

\section{Inclusion criteria}

In patients diagnosed with SCD, the inclusion criteria were based acute bone pain and chronic persistent bone pain with a clinical concern of osteomyelitis.

\section{Exclusion criteria}

Skeletal trauma and musculoskeletal pathologies other than SCD were the exclusion criteria.

\section{CSI and MRI techniques}

All MRI studies were performed on a 1.5-T Achieva; Philips (Netherlands) closed MRI machine and using appropriate surface receiver coils.

At least, the following sequences were performed in all studies:

1- Fat-suppressed fluid-sensitive sequence using either short tau inversion recovery STIR (TR/TE 6580/ 102, TI 130) or TSE T2-fs (TR/TE 4200/92 frequency selective fat saturation) or TSE PD-fs (TR/ TE 7650/34 frequency selective fat saturation).

2- Pre-contrast T1-fs sequence (TR/TE 883/23, frequency selective fat saturation).

3- T1-weighted dual gradient-echo in-phase and outof-phase sequence in axial and/or coronal planes (TR/TEs, 7/4.8 and 2.4).
4- Post-contrast T1-fs sequence (TR/TE 883/23), in more than one plane, in case of absence of $\mathrm{T} 1$ hyperintensity on pre-contrast images and after ensuring that the renal function is normal. $0.2 \mathrm{ml} / \mathrm{kg}$ body weight Magnevist (Schering, Germany) was used.

\section{Image analysis}

Three observers with 13, 6, and 5 years of experience interpreted the images independently. For diffuse marrow signal alteration, the IP and OP images were simultaneously analyzed for signal intensity; equal-sized region of interest (ROI) with equal number of pixels were placed over the same area of normal-appearing bone marrow (i.e., lacking any focal lesions) on the IP and the corresponding OP images. In order to perform an accurate measurement, the entire sequences were carefully scrutinized to avoid placement of the ROI cursors over any focal marrow abnormality, and the selected position for ROI placement was targeted on an area that did not attain any hyperintensity on STIR and/or T1WI. Computation of the percentage of signal loss on CSI was then obtained from IP-OP/IP formula; the result was considered abnormal when the value was $<20 \%$, absent, or shows negative value.

For focal bone marrow lesions whether osteonecrosis or osteomyelitis, the STIR sequence together with the pre- and post-contrast-enhanced T1-fs and CSI images were scrutinized for number, location, and signal characteristics of signal abnormality areas within the bone marrow and/or the overlying soft tissues.

The frequency of detection of marrow focal lesions was recorded across different study sequences in order to assess the value of IP and OP images in focal marrow abnormality detection.

\section{Statistical analysis}

Results of CSI were recorded and tabulated. The statistical analysis was done using SPSS-16, and the descriptive data were represented as number and percent. Comparison between serum ferritin levels across different patterns of diffuse bone marrow signal changes was done by ANOVA test. Correlation between serum ferritin level and the percentage of bone marrow signal loss on CSI was done and represented as a scatterplot. Interrater agreement was assessed for focal bone marrow abnormalities.

\section{Results}

Twenty males (47.6\%) and twenty-two females (52.4\%) who complained of bone pain were included in the study; their mean age was $26.95 \pm 9.1$ years (range 8-45 years). Thirty-five patients presented with acute bone pain, whereas seven patients had chronic bone pain. 


\section{Diffuse marrow abnormality findings}

There were four different patterns of diffuse bone marrow signal changes among all patients; they were (1) persistent red marrow with normal CSI percentage of signal loss (> 20\%) which suggest normal iron load, (2) diffuse abnormal hypointense patterns with either inadequate $(<$ $20 \%$ ) signal loss on OP, (3) diffuse abnormal hypointense patterns with no signal loss or signal reversal on OP (OP $>$ IP), and (4) diffuse signal void pattern on all sequences including IP and OP images. The patterns from 2 to 4 were suggestive of iron overload. Almost two-thirds of patients $(n=28,66.6 \%)$ had diffuse bone marrow signal abnormalities that suggest presence of iron overload; most of them (19 patients $\{\sim 45.23 \%\})$ showed lack of any signal loss on OP images (Table 1, Fig. 1).

Of the total, 30 patients $(71.4 \%)$ have a history of previous blood transfusions; however, no one was on chronic blood transfusion regimen nor had blood transfusion in the previous 2 months. Various ranges of serum ferritin level were found across different patterns of diffuse marrow changes; their means were increasing in accordance to the grade of marrow changes and the degree of signal changes found on CSI with statistically significant difference (Table 2)

On correlation analysis, there was significant high negative correlation between the percentage of signal loss on CSI obtained from IP-OP/IP formula and serum ferritin level (Fig. 2).

\section{Focal marrow abnormality findings}

Among all patients, there were 34 patients who had one or more focal marrow lesion with signal characteristics compatible with osteonecrosis (total number of lesions was 153); other 4 patients showed a focus of osteomyelitis (total number of lesions was 4). Final diagnostic confirmation for all lesions was based mainly on response to specific therapy and long-term follow-up. Bone biopsy confirmed all cases with osteomyelitis; this was performed surgically in two cases who underwent debridement, and by ultrasonographic-guided aspiration of subperiosteal and peri-osseous abscess in the two other cases.

The most common involved anatomic location by osteonecrosis was the femur notably the femoral head then the pelvic bones (Table 3, Fig. 3).
T1 hyperintensity on fat-suppressed $\mathrm{T} 1$ was diagnostic for osteonecrosis in acute pain setting; this was observed in 22 patients with total number of lesions was 83 lesions. Among the remaining 12 patients, 9 patients underwent post-contrast study and showed marginal enhancement of their focal marrow signal abnormalities (total number of lesions was 61); the imaging criteria for diagnosis of osteonecrosis in such cases were the presence of marginal enhancement, no patchy enhancement, no extra-osseous signal abnormality, no cortical defects and absent sequestration; final confirmed diagnosis was based on therapeutic response and long-term follow-up. The constant imaging feature in osteomyelitis lesions was the extra-osseous involvement and patchy enhancement (Table 4, Fig. 4).

Regarding CSI in focal marrow lesions, all T1 hyperintense lesions (83 lesions 54.2\%) demonstrated corresponding high signals on both IP and OP images. The frequency of focal marrow abnormality detection on CSI that attained STIR hyperintensity was relatively higher on OP compared with IP images (Table 5).

\section{Inter-rater reliability}

The percent agreement for focal marrow abnormalities was high; it was $98 \%$ for STIR high signal, $97 \%$ for OP signal abnormality, 95\% for T1 hyperintensity, and 91\% for IP images.

\section{Discussion}

Either diffuse or focal, bone marrow complications of SCD are common; they are related to chronic hemolysis, repeated blood transfusion, or vaso-occlusion [6].

Iron is toxic to bone marrow cells; it can be overloaded within the bone marrow in SCD especially in repeated blood transfusion. The toxicity of iron is accentuated by the effect of reactive oxygen species and hydroxyl radical leading to significant oxidant damage to the bone marrow cells and eventual ineffective erythropoiesis phenotype; the presence of such phenotype favors the development of iron cardiomyopathy; thus, the detection of iron overload in SCD is crucial $[7,8]$.

MRI has been utilized for non-invasive detection of iron overload in different tissue particularly the liver and the heart. The most simple and widely available method

Table 1 Diffuse bone marrow changes in SCD $(n=42)$

\begin{tabular}{|c|c|c|c|c|}
\hline & & Pattern & Number & Percent \\
\hline \multirow[t]{4}{*}{ Iron loading } & Overload (Hemosiderosis) & Diffuse hypointense pattern with no signal loss on OP & 19 & $\sim 45.23 \%$ \\
\hline & & Diffuse hypointense pattern with $<20 \%$ signal loss on OP & 6 & $\sim 14.3 \%$ \\
\hline & & Signal void pattern & 3 & $\sim 7.14 \%$ \\
\hline & No overload & Persistent red marrow with preserved signal loss on OP (> 20\%) & 14 & $\sim 33.33 \%$ \\
\hline Volume & & Diffuse marrow expansion & 2 & $\sim 4.8 \%$ \\
\hline
\end{tabular}




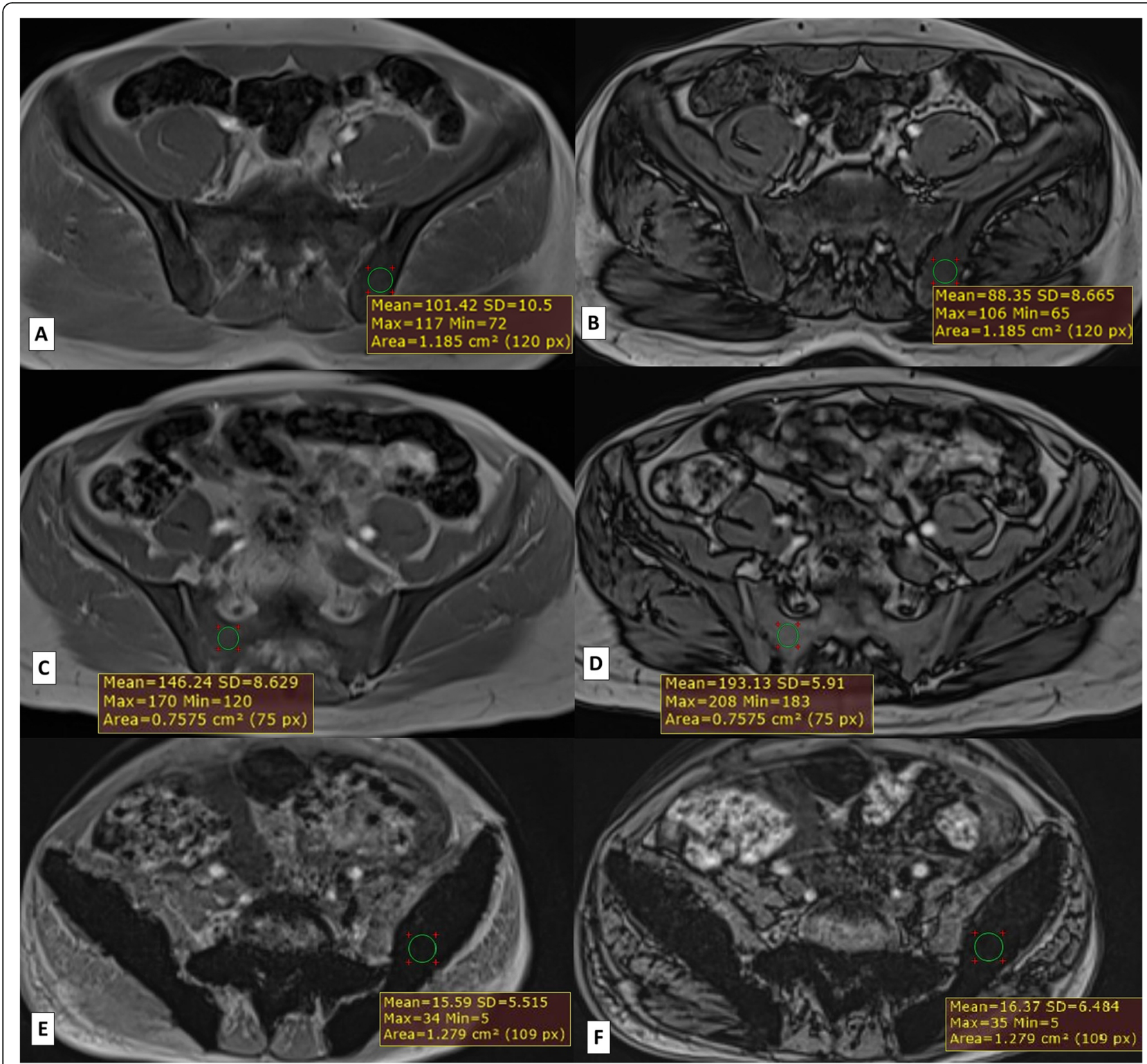

Fig. 1 Axial IP with corresponding OP images in three different patients with SCD and elevated serum ferritin; a and $\mathbf{b}$ show inadequate signal loss on OP images $<20 \%$, (IP-OP/IP $=12 \%)$, $\mathbf{c}$ and $\mathbf{d}$ show lack of normal signal loss with reversal of normal signal characteristics of CSI in a different patient, $\mathrm{OP}>\mathrm{IP},(\mathrm{IP}-\mathrm{OP} / \mathrm{IP}=-32 \%)$, e and $\mathbf{f}$ show signal void pattern with diffuse very low signal on both IP and OP images

Table 2 Serum ferritin level across different patterns of diffuse marrow changes

\begin{tabular}{lll}
\hline Pattern & Mean \pm SD & Range \\
\hline Persistent red marrow with preserved signal loss on OP $(>20 \%)$ & $189 \pm 94$ & $56-322$ \\
Diffuse hypointense pattern with $<20 \%$ signal loss on OP (OP $<$ IP) & $1054 \pm 499$ & $509-1765$ \\
Diffuse hypointense pattern with no signal loss on OP (OP > IP) & $1536 \pm 940$ & $568-3120$ \\
Signal void pattern & $3521 \pm 408$ & $3095-3910$ \\
\hline
\end{tabular}




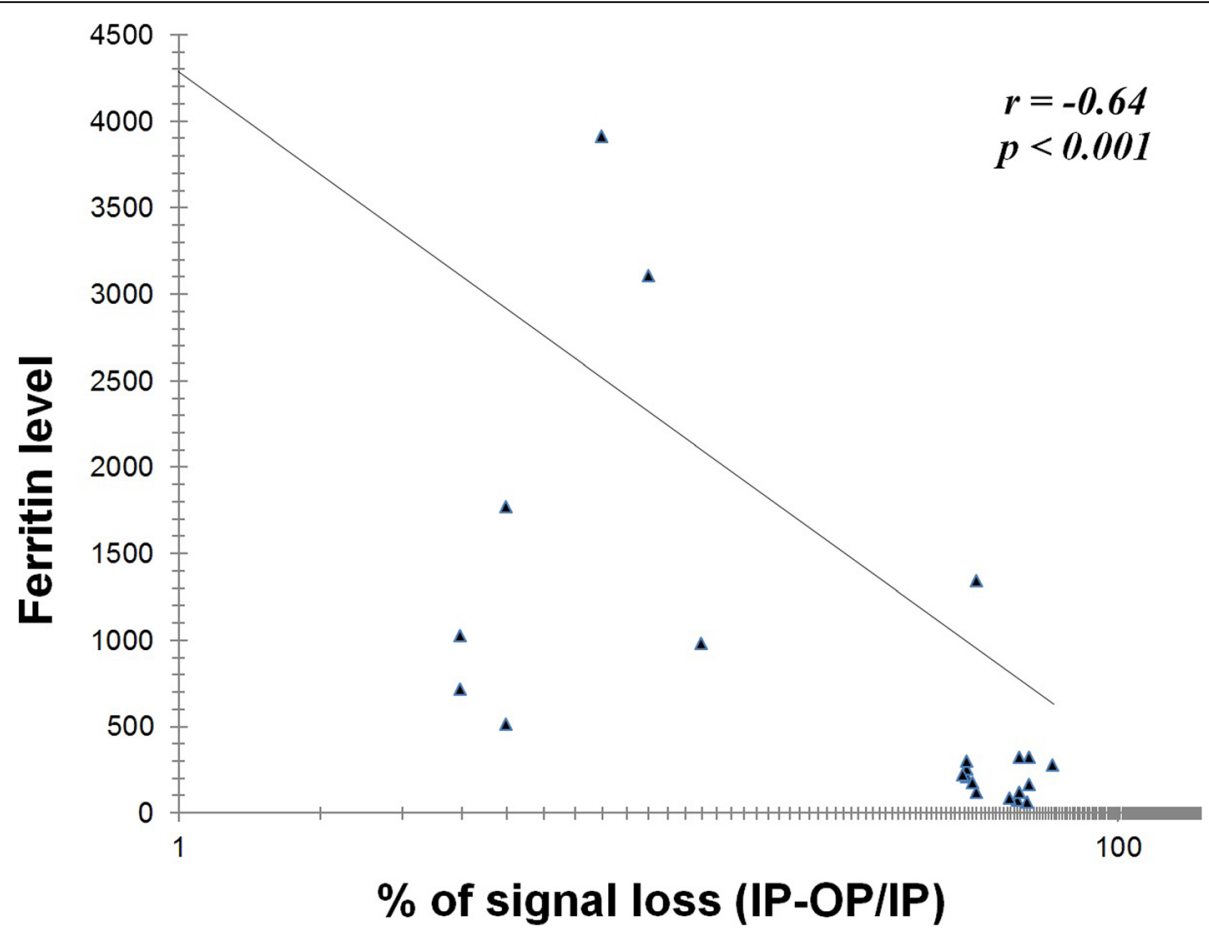

Fig. 2 Correlation scatterplot, expressed in a logarithmic scale, demonstrates significant high negative correlation between the serum ferritin level and the percentage of signal loss on CSI

is the liver-to-muscle signal intensity ratio which is based on iron-induced $\mathrm{T}^{*}$ s shortening. The current study utilized the same idea but on a different sequence; it depended on the hypothesis stated that the signal intensity decrease observed on IP images, compared with OP images, is related to iron-induced magnetic susceptibility and $\mathrm{T}_{2} \%$ shortening. And this is the novel point in the study, as there is no known report that studied marrow iron overload using CSI $[6,9,10]$.

Among different patterns of diffuse marrow signal changes found in the present study, $2 / 3$ of the patients showed evidence of iron overload in the form of abnormal percentage of signal loss $(<20 \%)$ on OP that was negatively correlated with serum ferritin level. This finding highlights the diagnostic use of CSI in iron detection in patients with SCD and could emphasize the value of adding such sequence to the routine skeletal MRI in SCD. Although a few reports studied the role of MRI in detecting bone marrow abnormality in hemoglobinopathy-related hemosiderosis, there is a paucity of studies concerning with the role of CSI in detection of iron overload in bone marrow or other tissues. Drakonaki et al. [11] studied

Table 3 Location of focal signal abnormality marrow lesions (number of cases $=38 / 42, \sim 90.5 \%$ )

\begin{tabular}{|c|c|c|c|}
\hline & Location & No. of lesions & Percent \\
\hline \multirow[t]{8}{*}{ Osteonecrosis (34/38 cases $\sim 89.5 \%)(n$. of lesions $=153$ ) } & Femoral head & 46 & $\sim 30.1 \%$ \\
\hline & Femoral shaft and trochanteric region & 43 & $\sim 28.1 \%$ \\
\hline & Pelvic bones & 31 & $\sim 20.2 \%$ \\
\hline & Sacral & 12 & $\sim 8 \%$ \\
\hline & Tibial & 5 & $\sim 3.3 \%$ \\
\hline & Fibular & 3 & $\sim 2 \%$ \\
\hline & Vertebral & 5 & $\sim 3.3 \%$ \\
\hline & Humeral & 8 & $\sim 5 \%$ \\
\hline \multirow[t]{3}{*}{ Osteomyelitis (4/ 38 cases $\sim 10.5 \%$ ) ( $n$. of lesions $=4$ ) } & Left femoral & 2 & $50 \%$ \\
\hline & Right tibial & 1 & $25 \%$ \\
\hline & Left tibial & 1 & $25 \%$ \\
\hline
\end{tabular}




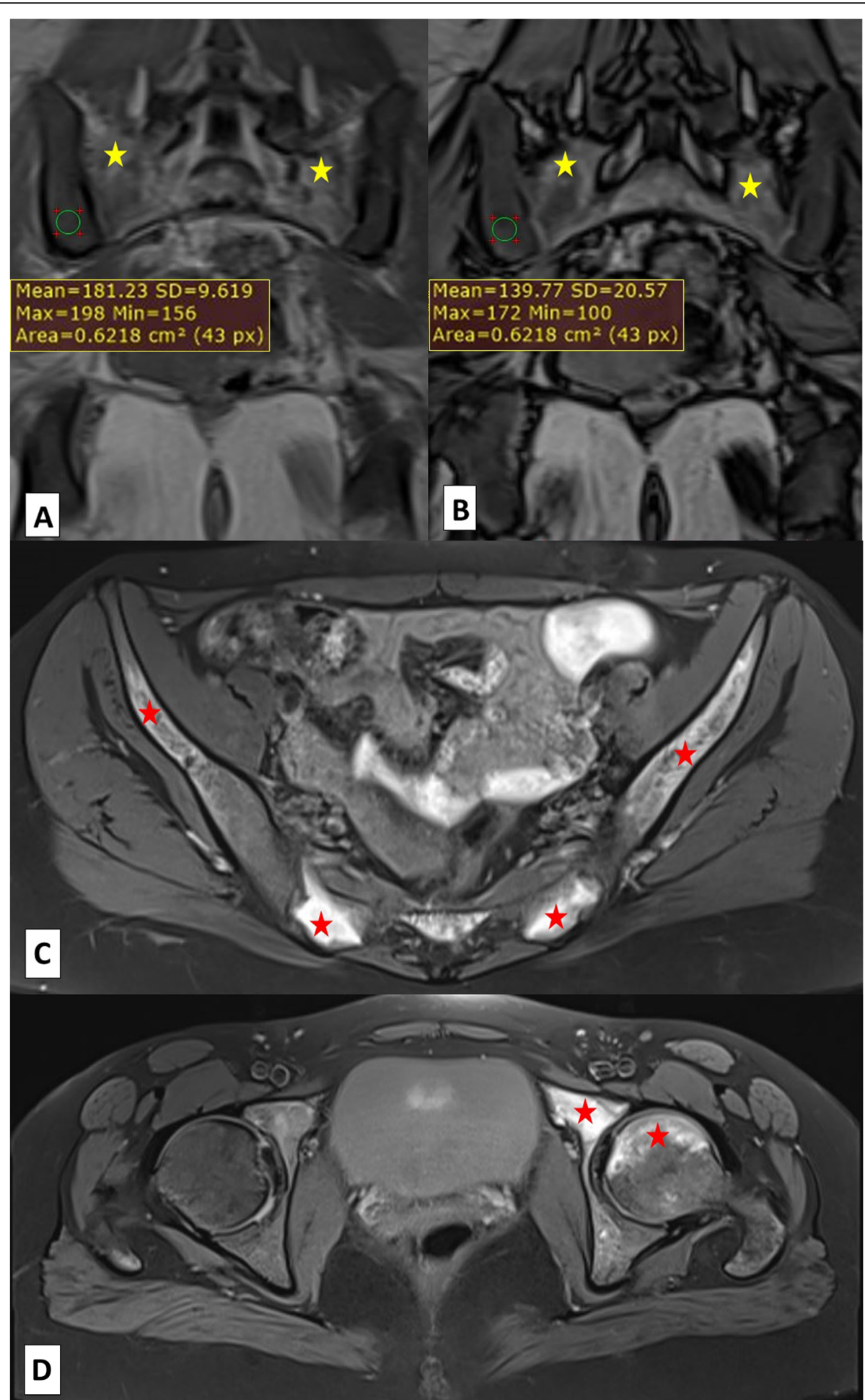

Fig. 3 Coronal IP (a) and OP (b) with axial T1fs $(\mathbf{c} \& \mathbf{d})$ in 27 year-old female patient with bilateral acute pelvic bone pain, show diffuse persistent red marrow with normal percentage of signal loss on OP (IP-OP/IP $=23 \%$ ), note the patient had diffuse low signals on T1 (not shown). (c \& $\mathbf{d}$ ) show multi-focal T1 hyperintensity in the sacrum, pelvic bone, left acetabulum and left femoral head consistent with acute osteonecrosis, note corresponding sacral hyperintensity on IP and OP (stars) 
Table 4 Imaging features of focal signal abnormality marrow lesions (number of cases = 38/42, 90.5\%)

\begin{tabular}{|c|c|c|c|}
\hline & Imaging feature & $\begin{array}{l}\text { No. of } \\
\text { lesions }\end{array}$ & Percent \\
\hline \multirow{4}{*}{$\begin{array}{l}\text { Osteonecrosis ( } 34 / 38 \text { cases } \sim 89.5 \%) \text { ( } n \text {. of lesions } \\
=153 \text { ) }\end{array}$} & Patchy STIR high signals (acute lesion) * & 105 & $\sim 63.6 \%$ \\
\hline & $\begin{array}{l}\text { Serpiginous STIR hyperintensity and central low signals of fat (chronic } \\
\text { lesion)* }\end{array}$ & 48 & $\sim 31.4 \%$ \\
\hline & $\mathrm{T} 1$ hyperintensity on $\mathrm{T} 1 \mathrm{fs}$ & 83 & $\sim 54.2 \%$ \\
\hline & Marginal enhancement ** & 61 & N/A \\
\hline \multirow{4}{*}{$\begin{array}{l}\text { Osteomyelitis ( } 4 / 38 \text { cases } \sim 10.5 \%) \text { ( } n \text {. of lesions }= \\
\text { 4) }\end{array}$} & Presence of extra-osseous signal abnormality & 4 & $100 \%$ \\
\hline & Presence of sequestra & 2 & $50 \%$ \\
\hline & Cortical defect & 3 & $75 \%$ \\
\hline & Patchy enhancement & 4 & $100 \%$ \\
\hline
\end{tabular}

*STIR high signals refer to edema-like signals on STIR or other fluid-sensitive fat suppressed images

${ }^{* *}$ Not all patients underwent post-contrast study

multiple MRI sequences including T1-w Gradient Echo (GRE) in assessment of bone marrow changes in betathalassemia and their relation to serum ferritin. They found diffuse marrow hypointensity in T1-w GRE with decreased bone marrow to paraspinous muscle signal intensity ratios in 23/40 patients (57.5\%) along with significant negative correlation with mean ferritin values $(r=-0.610$, $p=0.01$ ). Although Drakonaki et al. [12] did not utilize
CSI in their evaluation, the used T1-w GRE with its echo time (TE: 4) is the same utilized in IP images in the current study; thus, it resembles IP images in its T2* effect and subsequently its capability of detecting iron overload. Furthermore, Westphalen et al. [13] studied the role of opposed-phase MRI in hepatic iron deposition; they found that in patients with hepatic iron deposition there was significant signal intensity loss on the IP images; however,

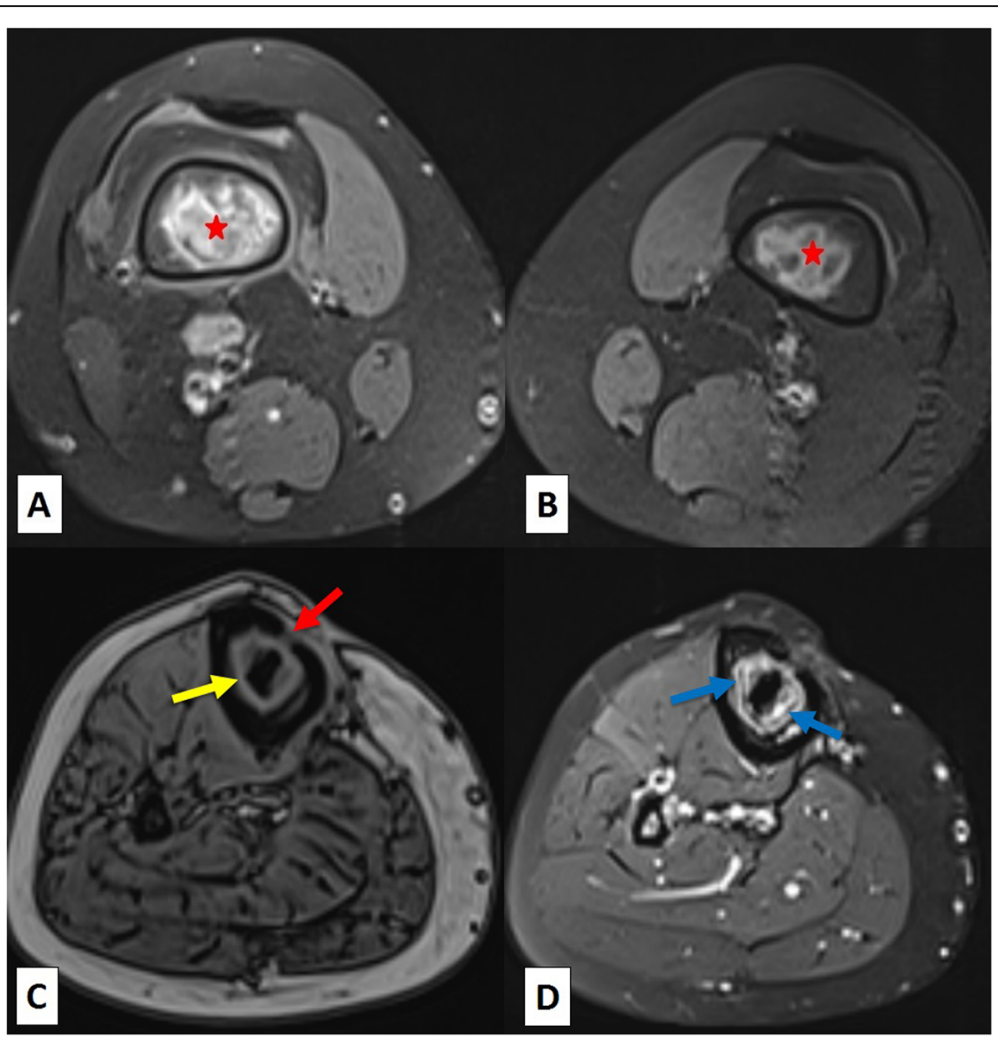

Fig. 4 Axial non-enhanced T1fs $\mathbf{a}$ and $\mathbf{b}$ through the lower femurs in 19-year-old male patient with bilateral acute thigh pain show bilateral focal bone marrow hyperintensity involving the right more than the left lower femoral shaft; features of acute marrow infarcts. Axial OP c and postcontrast $\mathrm{T} 1 \mathrm{fs} \mathbf{d}$ through the right upper tibial shaft on the same patient show focal intra-medullary hypointense sequestrum (yellow arrow), focal cortical interruption (red arrow), and non-homogenous marrow enhancement (blue arrows); features are consistent with chronic osteomyelitis 
Table 5 Frequency of focal marrow abnormality detection by CSI ( $n$. of lesions $=157$ )

\begin{tabular}{lll}
\hline Image series & No. of lesions & Percent \\
\hline Out of phase & 134 & $\sim 85 \%$ \\
In phase & 110 & $\sim 70 \%$ \\
\hline
\end{tabular}

they did not utilize the OP images in their evaluation nor correlate it with the serum ferritin level as the current study did [14, 15].

Regarding focal marrow lesions in the current study, the IP and OP images demonstrated high signal intensity in all cases of acute osteonecrosis that showed hyperintensity on T1fs images. This finding would be expected as both CSI and T1fs are T1 weighted; the ability of CSI to accurately detect $\mathrm{T} 1$ hyperintensity might extend its utility in SCD-related marrow abnormality especially if the T1fs suffered from artifact due to magnetic field inhomogeneity that commonly occurred on closed MR scanners, or there was lack of T1fs sequence for any reason such as in case of utilization of low field MRI [5].

The frequency of focal marrow abnormality detection on CSI that attained STIR hyperintensity was relatively higher on OP compared with IP images. Although this feature appears to have a little value especially if it stands alone against the ability of STIR in documentation of marrow edema-like lesions, it might bear the OP images a complementary role in lesion size detection, as lesion conspicuity is generally accentuated on OP images due to peripheral signal loss termed "India ink artifact" which is produced along the borders between fat and non-fatty tissue $[16,17]$.

\section{Conclusion}

CSI has high diagnostic performance in detecting diffuse marrow changes and development of iron overload in SCD. In SCD-related focal marrow lesions, CSI could have a complementary role in detection of $\mathrm{T} 1$ hyperintensity and lesion conspicuity.

\section{Abbreviations}

CSI: Chemical shift imaging; IP: In-phase; OP: Out-of-phase; ROI: Region of interest; SCD: Sickle cell disease

\section{Acknowledgements}

Not applicable.

\section{Authors' contributions}

AMA carried out data collection and imaging analysis. MFAS carried out study design and statistical and imaging analyses. MFAA carried out the statistical analysis and editing of publications/presentation in addition to imaging analysis. All authors read and approved the final manuscript.

\section{Funding}

The study had no funding from any resource.

\section{Availability of data and materials}

The datasets used and analyzed during the study are available from the corresponding author on reasonable request.

\section{Ethics approval and consent to participate}

The study was approved by the Research Ethics Committee of the Faculty of Medicine, Minia University, on 3 February 2019; reference number of approval: N/A. All cases gave written informed consent to participate in the research.

\section{Consent for publication}

All patients included in this study gave written informed consent for data publishing contained within this study.

\section{Competing interests}

The authors declare that they have no competing interests.

Received: 14 July 2020 Accepted: 13 December 2020

Published online: 11 January 2021

\section{References}

1. Piel FB, Williams TN (2016) Sickle cell anemia: history and epidemiology. In: Costa FF, Conran N (eds) Sickle cell anemia from basic science to clinical practice, vol 2, 1st edn. Springer International Publishing, Switzerland, pp 23-48

2. Patil SN, Veerappa K, Kiran R (2018) Avascular necrosis of the femoral head due to sickle cell disease in tribal population around Vishakhapatnam district, treated with core decompression and non-vascularised fibular graft. Int J Orthop Sci 4(3):672-676

3. Ejindu VC, Hine AL, Mashayekhi M et al (2007) Musculoskeletal manifestations of sickle cell disease. Radiographics 27(4):1005-1021

4. Coates TD, Wood JC (2017) How we manage iron overload in sickle cell patients. Br J Haematol. 177(5):703-716

5. Jain R, Sawhney S, Rizvi SG (2008) Acute bone crises in sickle cell disease: the T1 fat-saturated sequence in differentiation of acute bone infarcts from acute osteomyelitis. Clin Radiol 63(1):59-70

6. Queiroz-Andrade $M$, Blasbalg R, Ortega CD et al (2009) MR imaging findings of iron overload. RadioGraphics 29:1575-1589

7. Kosaraju V, Harwani A, Partovi S et al (2007) Imaging of musculoskeletal manifestations in sickle cell disease patients. Br J Radiol. 90(1073):20160130. https://doi.org/10.1259/bjr.20160130

8. Breda L, Rivella S (2014) Modulators of erythropoiesis: emerging therapies for hemoglobinopathies and disorders of red cell production. Hematol Oncol Clin North Am. 28:375-386

9. Camaschella C, Nai A (2016) Ineffective erythropoiesis and regulation of iron status in iron loading anaemias. Br J Haematol. 172:512-523

10. Labranche R, Gilbert G, Cerny M et al (2018) Liver iron quantification with MR imaging: a primer for radiologists. RadioGraphics 38:392-412

11. Moulopoulos LA, Koutoulidis $V$ (2015) The abnormal bone marrow: MRI patterns. In: Moulopoulos LA, Koutoulidis V. eds. Bone marrow MRI: a pattern-based approach. 1st ed. Springer-Verlag Italia (4):35-56. DOI. https:// doi.org/10.1007/978-88-470-5316-8 1

12. Meloni A, Renni R, Romano N, et al. (2014) Quantitative T2*MRI for bone marrow iron overload assessment in thalassemia major and intemedia patients. Blood 124 (21): 4042. https://doi.org/10.1182/blood.V124.21.4042. 4042

13. Levin TL, Sheth SS, Hurlet A et al (1995) MR marrow signs of iron overload in transfusion-dependent patients with sickle cell disease. Pediatr Radiol 25: 614-619

14. Drakonaki EE, Maris TG, Papadakis A et al (2007) Bone marrow changes in beta-thalassemia major: quantitative MR imaging findings and correlation with iron stores. Eur Radiol 17:2079-2087. https://doi.org/10.1007/s00330006-0504-y

15. Westphalen AC, Qayyum A, Yeh BM et al (2007) Liver fat: effect of hepatic iron deposition on evaluation with opposed-phase MR imaging. Radiology 242(2):450-455

16. Pezeshk P, Alian A, Chhabra A (2017) Role of chemical shift and Dixon based techniques in musculoskeletal MR imaging. EJ Radiol 94:93-100

17. Mohey N, Hassan TA (2019) Feasibility of MRI in early diagnosis of musculoskeletal complications of sickle cell disease in pediatrics. Med J Cairo Univ. 87(5):2693-2706

\section{Publisher's Note}

Springer Nature remains neutral with regard to jurisdictional claims in published maps and institutional affiliations. 